\title{
A descriptive study of ciguatera fish poisoning in Cook Islands dogs and cats: Treatment and outcome
}

\author{
Michelle J. Gray ${ }^{10}$ and M. Carolyn Gates ${ }^{2}$ (D) \\ 1. Master of Veterinary Medicine Program, School of Veterinary Science, Massey University, Palmerston North, \\ New Zealand; 2. EpiCentre, School of Veterinary Science, Massey University, Palmerston North, New Zealand. \\ Corresponding author: Michelle J. Gray, e-mail: mail.mgray@gmail.com \\ Co-author: MCG: c.gates@massey.ac.nz \\ Received: 01-02-2020, Accepted: 12-05-2020, Published online: 06-07-2020
}

doi: www.doi.org/10.14202/vetworld.2020.1269-1279 How to cite this article: Gray MJ, Gates MC (2020) A descriptive study of ciguatera fish poisoning in Cook Islands dogs and cats: Treatment and outcome, Veterinary World, 13(7): 1269-1279.

\begin{abstract}
Background and Aim: Ciguatera fish poisoning (CFP) is an illness caused by the ingestion of fish containing ciguatoxins. Dogs and cats are susceptible to CFP, but there is little published and much unknown about the condition in these species. This study aimed to document the treatment and outcome of canine and feline cases of CFP, and to look for prognostic indicators.

Materials and Methods: Six years of medical records from the Esther Honey Foundation Animal Clinic (the only veterinary clinic in the Cook Islands during the study period) were reviewed to identify cases of CFP. Data relating to treatment and outcome were collected.

Results: Two hundred and forty-six cases of CFP were identified, comprising 165 dogs and 81 cats. The treatments most commonly administered to cases were fluid therapy and muscle relaxants. Mannitol was only given to five animals. The survival rate was $>90 \%$ and almost all mortalities occurred in the first week of hospitalization. Recovery was slow, with hospitalization averaging 12.9 days. There was no significant difference in recovery times between dogs and cats. Prolonged periods of anorexia and recumbency were common in both species. Factors associated with prolonged recovery times included case severity, anorexia, and age (in dogs).
\end{abstract}

Conclusion: This article documented the treatment and outcome of animals afflicted by CFP in the Cook Islands. Therapy for CFP was primarily symptomatic and supportive. The survival rate was high, but recovery was often prolonged. The findings will assist veterinarians in giving prognoses and managing owner expectations.

Keywords: cats, ciguatera, Cook Islands, dogs, outcome, treatment.

\section{Introduction}

Ciguatera fish poisoning (CFP) is an illness caused by the ingestion of naturally occurring ciguatoxins. The toxins are produced by microalgae and transferred through the marine food chain. Poisoning occurs after the ingestion of ciguatoxin containing fish [1-3]. Dogs and cats are among the species afflicted by CFP. Indeed, last century, both species were used in bioassays and as test subjects for research into CFP [4-9]. More recently, there have been case reports [10-15], and general articles about the toxicity [16-22]. Objective studies of CFP in dogs and cats are, however, lacking.

The clinical presentation of CFP varies between species [23]. Human CFP is characterized by a combination of gastrointestinal, cardiovascular, and sensory abnormalities [1,2,24]. In contrast, CFP in dogs and cats is characterized by motor impairment, and in

Copyright: Gray and Gates. Open Access. This article is distributed under the terms of the Creative Commons Attribution 4.0 International License (http://creativecommons.org/licenses/ by/4.0/), which permits unrestricted use, distribution, and reproduction in any medium, provided you give appropriate credit to the original author(s) and the source, provide a link to the Creative Commons license, and indicate if changes were made. The Creative Commons Public Domain Dedication waiver (http:// creativecommons.org/publicdomain/zero/1.0/) applies to the data made available in this article, unless otherwise stated. particular, by ataxia and paresis $[4,7,18]$. A detailed description of the symptomology of canine and feline CFP in this case series was presented in an earlier article [25].

There is no specific antidote for ciguatoxin, which acts by binding to voltage sensitive sodium channels [26-28]. Treatment of CFP is, therefore, mostly symptomatic and supportive. Many drugs have been used empirically in human medicine to alleviate gastrointestinal, neurologic, and cardiovascular symptoms [1]. Mannitol is also widely recommended [1] since Palafox et al. [29] described the reversal of neurologic symptoms of CFP after mannitol administration. Controlled trials of mannitol therapy for CFP have, however, produced conflicting results and reviewers have argued both for and against its efficacy [30-32].

Treatments for canine and feline CFP have largely been extrapolated from human medicine. Those reported in the literature have included thiamine [14]; steroids, Vitamin B, and "cardiovascular agents" [4]; and atropine, calcium, and lignocaine [9]. However, most of the veterinary case reports attribute patient recovery primarily to nursing care and nutritional support $[11,12,16,18]$. The treatment protocol of the Esther Honey Foundation (EHF) Animal Clinic (the site of 
this study) recommends: Decontamination if possible (with emesis and/or activated charcoal), fluid therapy, nutritional support, muscle relaxants, mannitol, nursing care, and symptomatic medication as indicated (e.g., analgesics and gastrointestinal medications) [18].

The prognosis for human CFP is good. Although the toxicity can be debilitating, overall mortality rates are estimated to be $<0.1 \%[33,34]$. Fatalities, when they occur, are due to dehydration, cardiovascular shock, or respiratory failure [1,2]. Recovery times can vary from days to months $[2,35]$. Sensitization can occur after acute CFP in people. This is a phenomenon whereby patients experience a recurrence or aggravation of symptoms following the ingestion of non-toxic fish, food, or drinks. The cause of sensitization is unknown, but hypotheses include bioaccumulation of ciguatoxin and neurologic sensitization [1].

The prognosis for dogs and cats with CFP appears similarly good. While deaths have been reported in animals [13], the majority of (non-experimental) cases have recovered $[10-12,14,15]$. The duration of illness in dogs and cats is reported to be 1-3 weeks (Table-1). It is unknown whether CFP sensitization occurs in dogs and cats.

This study is the first to specifically evaluate the treatment and outcome of canine and feline CFP cases. A review of current treatment practices is important, both to inform veterinarians faced with treating CFP, and to identify therapies worthy of further investigation. Relying on treatment data from human studies is not appropriate, given the species differences in symptomology. The objective examination of survival rates and recovery times for canine and feline CFP cases is also important, as it will enable veterinarians to give more accurate, evidence-based prognoses for afflicted animals.

This article is the third in a series describing CFP in dogs and cats in the Cook Islands. It aims to describe the treatment and outcome of CFP cases. A second objective is to identify potential prognostic indicators.

\section{Materials and Methods}

\section{Ethical approval}

This retrospective review of case records was deemed to not require ethics approval (Massey University).

Table-1: Duration of illness in reports of canine and feline ciguatera fish poisoning.

\begin{tabular}{lll}
\hline Reference & Species & $\begin{array}{l}\text { Reported time } \\
\text { to recovery }\end{array}$ \\
\hline Anonymous [10] & Dogs & 2 weeks \\
Cohen [16] & Dogs & $2-3$ weeks \\
Forster [18] & Dogs + Cats & Weeks \\
Bagnis and Fevai [4] & Cats & $7-10$ days \\
Hessel et al. [6] & Cats & $1-5$ days \\
Clark and Whitwell [11] & Cats & $2+$ weeks \\
Kemppainen et al. [12] & Cats & 10 days \\
Seawright [22] & Cats & $2-14$ days \\
Tonge [15] & Cats & $2-7$ days \\
\hline
\end{tabular}

\section{Data collection}

The location and methodology of this study is described in detail elsewhere [36]. In brief, the medical records of the EHF Animal Clinic (the only veterinary clinic in the Cook Islands during the study period) were searched for cases with a presumptive diagnosis of CFP. Cases presenting in the 6-year period March 2011-February 2017 were considered for inclusion. Eligible patient files were searched to identify the variables of interest: Treatments administered and details of case outcome (Supplementary Table-1). Data relating to case demographics, exposure history, and clinical signs were also collected $[25,36]$ and used in the survival analyses. Data were collated using EpiInfo software (version 7.2.1.0, CDC, Atlanta, USA).

The age variable was assigned categorical values based on the following criteria:

Juvenile: Age given as $\leq 12$ months; OR animal referred to as a puppy or kitten

Adult: Age given as $>12$ months and $<8$ years; OR animal referred to as an adult

Senior: Age given as $\geq 8$ years; $O R$ animal referred to as senior, aged or geriatric

Unspecified: Insufficient detail in the medical record to classify the case as juvenile, adult, or senior

Severity of locomotor dysfunction was classified as mild (no locomotor signs, or ataxia or paresis without recumbency) or severe (sternal or lateral recumbency).

Severity of respiratory dysfunction was classified as mild (no respiratory signs or tachypnea) or severe (moderate or marked dyspnea).

\section{Statistical analysis}

Days anorexic, days recumbent, and days in hospital were calculated by subtracting the presenting date from the date a case started eating (days anorexic); starting walking (days recumbent); and was discharged or died (days in hospital).

Descriptive statistics were performed in EpiInfo. Using the subset of 207 surviving cases with known discharge dates, Kaplan-Meier curves were plotted, and a log-rank test was performed to determine if there was a statistically significant difference in the time to discharge between dogs and cats presumptively diagnosed with CFP. For each species, a survival analysis was then performed using a Cox Proportional Hazards model to identify clinical signs and case characteristics that were associated with the time until discharge. Each variable was screened in a univariate analysis to identify associations with $\mathrm{p}<0.20$ for inclusion in the final multivariable model. A backwards stepwise selection procedure was then used to sequentially remove variables with the highest $p$-value until all remaining variables in the multivariable model had $\mathrm{p}<0.05$. The significant associations from the multivariable model were reported as hazards ratios with $95 \%$ confidence intervals. 


\section{Results}

Two hundred and forty-six cases with a presumptive diagnosis of CFP were identified from the 6 -year pool of medical records. These comprised of 165 dogs and 81 cats.

\section{Treatment}

Fifteen cases (ten dogs and five cats) received no treatment other than hospitalization, observation, and flea/worm medication. Two hundred and thirty-one cases received one or more treatments. The most common therapies administered were fluid therapy and muscle relaxants. Mannitol was the least frequent treatment, with only five cases (four dogs and one cat) receiving an infusion. Table-2 documents the type and frequency of treatments given in this case series.

\section{Outcome}

Outcome was specified in 236 cases (95.9\%). Five dogs and five cats had no outcome recorded. Of the cases with known outcome, 216 survived (91.5\%); 12 died (5.1\%); and eight were euthanized (3.4\%). Table-3 provides a breakdown of outcome by species.

Cause of death was recorded for seven of the 12 cases that died. Five deaths (three dogs and two cats) were due to respiratory arrest (with aspiration suspected in three cases). One cat died during anesthesia for esophageal tube placement; and one dog died from hemorrhagic gastroenteritis (after apparent resolution of CFP).

Reasons given for euthanasia included lack of improvement (one dog and two cats); deterioration (one dog and one cat); and poor clinical state (two dogs). No reason was given for euthanasia of one cat.

\section{Duration of illness}

The duration of hospitalization could be calculated for 207 of the 216 surviving cases (95.8\%). Case records of six dogs and three cats failed to specify the date of discharge/recovery. The mean duration of hospitalization was 12.9 days and the median duration 10 days. The longest recorded hospitalization in a dog was 63 days, versus 47 days for a cat. Overall, dogs in this case series recovered slightly quicker (mean 12.3 days, median 9 days, $n=142$ ) than cats (mean 14.3 days, median 12 days, $n=65$ ). However, when Kaplan-Meier curves were plotted (Figure-1), the difference was not statistically significant (log-rank test, $p=0.258$ ). Figure- 2 depicts the range of recovery times.

When cases were graded by the degree of locomotor and respiratory dysfunction, severely affected cases took longer on average to recover (Table-4). The difference was most apparent when comparing dogs with mild versus severe locomotor dysfunction.

This observation was confirmed by a survival analysis for canine CFP, which identified recumbency, rigidity, and paresis as hazards for prolonged hospitalization. Other clinical signs associated with prolonged illness included dehydration, anorexia, seizures, and hyperesthesia/dysesthesia. The predictive model for time to discharge of canine CFP cases also retained age as a hazard. When compared with the reference group of adult dogs, cases described as senior were $82 \%$ less likely to be discharged after any particular duration of hospitalization. Hazard ratios, 95\% confidence intervals, and $\mathrm{p}$ values are presented in Table-5.

Survival analysis for feline CFP identified tremors, anorexia, and cardiac irregularities as hazards for prolonged illness. Of these, the magnitude of the effect was greatest for cases with cardiac irregularities. Fish exposure was also retained in the predictive model for time to discharge in feline CFP. Compared to the reference group of cats without known fish ingestion,

Table-2: Treatments administered to ciguatera fish poisoning cases.

\begin{tabular}{|c|c|c|c|}
\hline Treatment type & $\begin{array}{l}\text { Number of } \\
\text { cases }\end{array}$ & $\begin{array}{l}\text { \% of cases } \\
(n=246)\end{array}$ & Specific treatment (number of cases) \\
\hline Fluids & 200 & 81.3 & Intravenous fluids (189); subcutaneous fluids (29); oral fluids (17) \\
\hline Muscle relaxants & 123 & 50.0 & Diazepam (117); methocarbamol (39); midazolam (6) \\
\hline Other $^{1}$ & 100 & 40.6 & $\begin{array}{l}\text { Maropitant (16), metoclopramide (16), atropine }(12) \text {, ranitidine }(6) \text {, } \\
\text { cyproheptadine }(6) \text {, other }(54)^{2}\end{array}$ \\
\hline Nutritional support & 89 & 36.2 & Assisted/syringe feeding ( 84$)$; nasogastric tube (10); esophageal tube (3) \\
\hline Analgesics & 74 & 30.1 & Buprenorphine (32); morphine (29); butorphanol (19); tramadol (6) \\
\hline Decontamination & 62 & 25.2 & Activated charcoal (60); emesis (4) \\
\hline Supplements & 58 & 23.6 & Potassium (29); B vitamins (22); glucose $(10)$; other $(15)^{3}$ \\
\hline Antibiotics & 51 & 20.7 & $\begin{array}{l}\text { Amoxiclav (23), amoxicillin (9), cephalexin (8), enrofloxacin (6), } \\
\text { metronidazole/spiramycin (6), other (11) }\end{array}$ \\
\hline NSAIDs & 25 & 10.2 & Meloxicam (14), carprofen (5) \\
\hline Sedatives & 20 & 8.1 & Acepromazine (19); Phenobarbitone (3) \\
\hline Eye ointment & 10 & 4.1 & Lubricant (5), various antibiotic ointments (5) \\
\hline Steroids & 9 & 3.7 & Dexamethasone (7), prednisolone (2) \\
\hline Mannitol & 5 & 2.0 & \\
\hline
\end{tabular}


cats that were fed fish by their owners were 3 times more likely to be discharged after any particular duration of hospitalization. Hazard ratios, $95 \%$ confidence intervals, and $\mathrm{p}$ values are presented in Table-6.

Recovery times for animals who had repeated episodes of CFP are detailed in Table-7. There was no consistent pattern of either slower or faster recovery during their latter episodes of illness.

Table-3: Outcome of ciguatera fish poisoning cases.

\begin{tabular}{lccc}
\hline Outcome & $\begin{array}{c}\text { Canine and } \\
\text { feline cases (\%) }\end{array}$ & $\begin{array}{c}\text { Canine cases } \\
\mathbf{( \% )}\end{array}$ & $\begin{array}{c}\text { Feline } \\
\text { cases (\%) }\end{array}$ \\
\hline Survived & $216(87.8)$ & $148(89.7)$ & $68(84.0)$ \\
Died & $12(4.9)$ & $8(4.8)$ & $4(4.9)$ \\
Euthanized & $8(3.3)$ & $4(2.4)$ & $4(4.9)$ \\
Unknown & $10(4.1)$ & $5(3.0)$ & $5(6.2)$ \\
Total & $246(100.0)$ & $165(100.0)$ & $81(100.0)$ \\
\hline
\end{tabular}

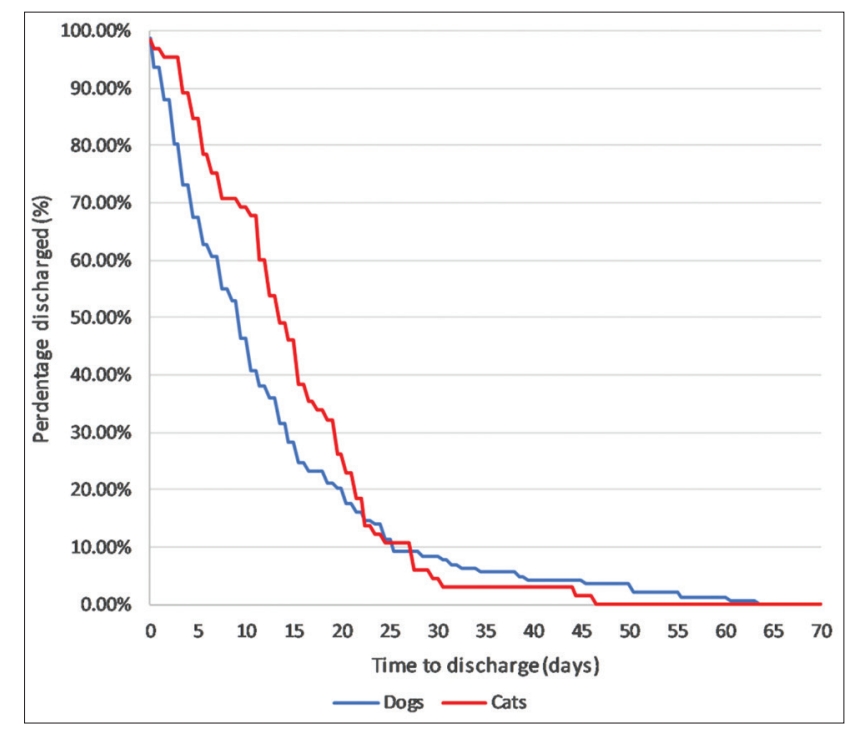

Figure-1: Kaplan-Meier survival curve for time to discharge in dogs and cats with ciguatera fish poisoning.

\section{Duration of anorexia and recumbency}

One hundred and thirty-three cases had anorexia (55.9\% of 238 with recorded clinical signs). It took on average 6.1 days for anorexic animals to start eating voluntarily; the range was 0-32 days. This is based on the data from 116 cases: Data were missing for eight animals that died, and another nine records did not specify the date that eating resumed. Figure- 3 depicts the duration of anorexia across all cases. Species differences in the duration of anorexia are presented in Table-8.

One hundred and forty-seven cases were recumbent at some stage of hospitalization $(61.8 \%$ of 238 with recorded clinical signs). It took on average 11.7 days for recumbent animals to stand and walk, and the range was $0-38$ days. This is based on the data from 107 cases: Data were missing for 13 animals that died, and another 27 records did not specify the date the animal start walking. Figure- 4 depicts the duration of recumbency across all cases. Species differences in the duration of recumbency are presented in Table-8.

\section{Time to death or euthanasia}

For the 12 cases that died, the mean time to death was 4.5 days (median 3.5 days, range 0-18 days). Only one case (a dog that died from hemorrhagic gastroenteritis) survived longer than a week. All others died in $\leq 7$ days.

For the eight cases that were euthanized, then mean time to euthanasia was 5.0 days (median 4.5 days, range 0-13 days). Only one case (a cat for whom no reason for euthanasia was given), survived longer than a week. All others were euthanized in $\leq 7$ days.

\section{Discussion}

\section{Study limitations}

The results of this study should be interpreted in light of the methodology. Classification of cases

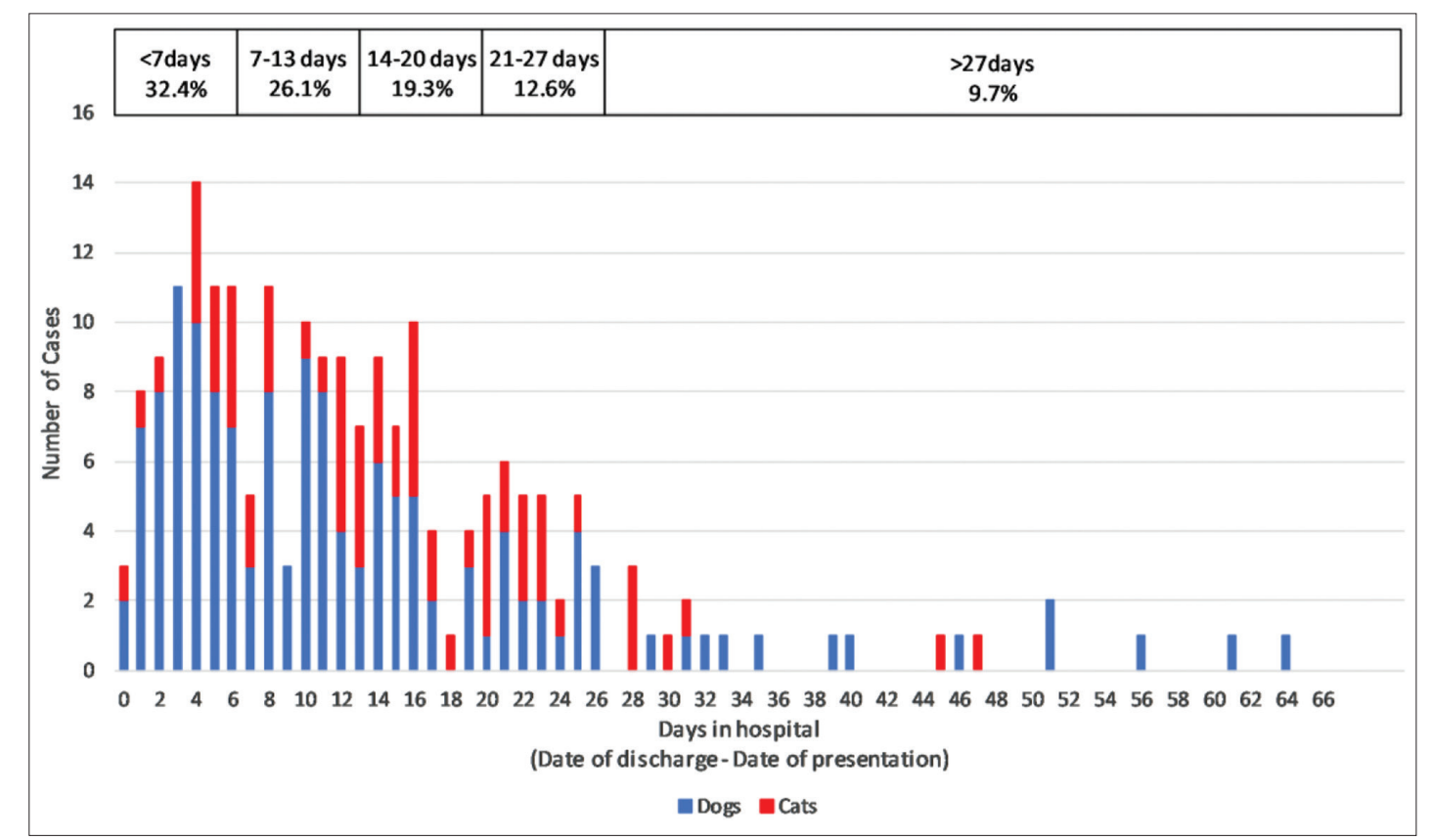

Figure-2: Duration of hospitalization of canine and feline cases of ciguatera fish poisoning: Data from 207 surviving cases. 
Table-4: Duration of hospitalization: Comparison of cases with mild versus severe locomotor and respiratory dysfunction.

\begin{tabular}{|c|c|c|c|c|c|}
\hline \multirow[t]{2}{*}{ Population } & \multirow[t]{2}{*}{ Classification } & \multirow[t]{2}{*}{ Number of cases } & \multicolumn{3}{|c|}{ Days in hospital } \\
\hline & & & Mean & Median & Range \\
\hline \multicolumn{6}{|c|}{ Degree of locomotor dysfunction } \\
\hline \multirow[t]{2}{*}{ All cases } & Mild $^{1}$ & 88 & 6.6 & 5 & $0-23$ \\
\hline & Severe $^{2}$ & 119 & 17.6 & 15 & $0-63$ \\
\hline \multirow[t]{2}{*}{ Dogs } & Mild $^{1}$ & 62 & 5.0 & 3 & $0-19$ \\
\hline & Severe $^{2}$ & 80 & 17.9 & 15 & $0-63$ \\
\hline \multirow[t]{2}{*}{ Cats } & Mild $^{1}$ & 26 & 10.4 & 10 & $0-23$ \\
\hline & Severe $^{2}$ & 39 & 16.9 & 16 & $1-47$ \\
\hline \multicolumn{6}{|c|}{ Degree of respiratory dysfunction } \\
\hline \multirow[t]{2}{*}{ All cases } & Mild $^{3}$ & 156 & 11.8 & 10 & $0-63$ \\
\hline & Severe $^{4}$ & 51 & 16.2 & 14 & $1-51$ \\
\hline \multirow[t]{2}{*}{ Dogs } & Mild $^{3}$ & 98 & 11.1 & 8 & $0-63$ \\
\hline & Severe $^{4}$ & 44 & 15.0 & 11.5 & $1-51$ \\
\hline \multirow[t]{2}{*}{ Cats } & Mild $^{3}$ & 58 & 13.1 & 12 & $0-45$ \\
\hline & Severe $^{4}$ & 7 & 24.0 & 23 & $8-47$ \\
\hline
\end{tabular}

${ }^{1}$ Mild=No locomotor signs, ataxia, or paresis without recumbency. ${ }^{2}$ Severe=Sternal or lateral recumbency. ${ }^{3} \mathrm{Mild}=\mathrm{No}$ respiratory signs or tachypnea. ${ }^{4}$ Severe=Moderate or marked dyspnea

Table-5: Cox proportional hazard estimates of the determinants of time to discharge in canine ciguatera fish poisoning cases.

\begin{tabular}{|c|c|c|c|c|}
\hline Variable & $\begin{array}{l}\text { Hazards } \\
\text { Ratio }\end{array}$ & $\begin{array}{c}\text { 95\% Confidence } \\
\text { interval }\end{array}$ & p-value & Mean duration of hospitalization \\
\hline Clinical signs $^{1}$ & & & & Cases with/without the clinical sign \\
\hline Recumbency & 0.3499 & $0.226-0.542$ & 0.000003 & 17 days/5 days \\
\hline Extensor rigidity & 0.4476 & $0.292-0.687$ & 0.0002 & 18 days/7 days \\
\hline Paresis & 0.537 & $0.368-0.783$ & 0.001 & 15 days/9 days \\
\hline Dehydration & 0.3652 & $0.186-0.716$ & 0.003 & 20 days/12 days \\
\hline Anorexia & 0.5643 & $0.379-0.841$ & 0.005 & 16 days/ 8 days \\
\hline Hyperesthesia/dysesthesia & 0.3296 & $0.130-0.833$ & 0.019 & 27 days/12 days \\
\hline Seizures & 0.4865 & $0.237-0.996$ & 0.049 & 24 days/11 days \\
\hline Dog age $^{2}$ & & & & By age group \\
\hline (Adult) $^{3}$ & 1.00 & - & - & 13 days \\
\hline Senior ${ }^{4}$ & 0.1773 & $0.048-0.649$ & 0.009 & 29 days \\
\hline Unspecified $^{5}$ & 0.5993 & $0.048-0.649$ & 0.068 & 13 days \\
\hline Juvenile ${ }^{6}$ & 1.0076 & $0.573-1.772$ & 0.979 & 9 days \\
\hline
\end{tabular}

${ }^{1}$ Documented at any stage during illness. ${ }^{2}$ Reference category in parenthesis. ${ }^{3}$ Adult: Age given as $>12$ months and $<8$ years; OR animal referred to as an adult. ${ }^{4}$ Senior: Age given as $\geq 8$ years; OR animal referred to as senior, aged or geriatric. ${ }^{5}$ Unspecified: Insufficient detail in medical record to classify case as juvenile, adult, or senior. ${ }^{6}$ Juvenile: Age given as $\leq 12$ months; OR animal referred to as a puppy or kitten

Table-6: Cox proportional hazard estimates of the determinants of time to discharge in feline ciguatera fish poisoning cases.

\begin{tabular}{|c|c|c|c|c|}
\hline Variable & $\begin{array}{l}\text { Hazards } \\
\text { Ratio }\end{array}$ & $\begin{array}{c}95 \% \text { Confidence } \\
\text { interval }\end{array}$ & p-value & Mean duration of hospitalization \\
\hline Clinical signs ${ }^{1}$ & & & & Cases with/without the clinical sign \\
\hline Tremors & 0.4491 & $0.246-0.819$ & 0.009 & 18 days $/ 13$ days \\
\hline Anorexia & 0.4927 & $0.287-0.847$ & 0.010 & 17 days $/ 11$ days \\
\hline Cardiac irregularities & 0.31 & $0.118-0.811$ & 0.017 & 21 days/14 days \\
\hline Source of exposure ${ }^{2}$ & & & & By source of exposure \\
\hline (No documented fish exposure) & 1.00 & - & - & 15 days \\
\hline Fish obtained through neighbor ${ }^{3}$ & NA & NA & NA & 0 days \\
\hline Fish obtained through owner & 3.6004 & $1.727-7.506$ & 0.0006 & 11 days \\
\hline Fish scavenged ${ }^{3}$ & 1.3209 & $0.307-5.691$ & 0.708 & 12 days \\
\hline Fish source unspecified ${ }^{3}$ & 0.5167 & $0.117-2.281$ & 0.383 & 28 days \\
\hline
\end{tabular}

${ }^{1}$ Documented at any stage during illness. ${ }^{2}$ Reference category in parenthesis. ${ }^{3}$ These groups contain $\leq 3$ cases

was based solely on the attending clinician's opinion. Therefore, some cases may have been erroneously attributed to CFP, and if so, the recovery and outcome data will be imprecise. Data collection was retrospective, and incomplete cases were included on the grounds that they still contained potentially valuable information. Consequently, the size of some data sets and the statistical power of some analyses are reduced. In addition, the frequency of treatments may be under-reported. A final consideration is that days in hospital were taken to reflect the duration of illness, as it was a measure that could be determined objectively. 
Table-7: Recovery times of animals with repeated episodes of ciguatera fish poisoning.

\begin{tabular}{llclccc}
\hline Species & $\begin{array}{l}\text { Date of CFP } \\
\mathbf{1}^{\text {st }} \text { episode }\end{array}$ & $\begin{array}{c}\text { Duration of } \\
\text { hospitalization } \\
\text { (days) }\end{array}$ & $\begin{array}{l}\text { Date of CFP } \\
\mathbf{2}^{\text {nd }} \text { episode }\end{array}$ & $\begin{array}{c}\text { Duration of } \\
\text { hospitalization } \\
\text { (days) }\end{array}$ & $\begin{array}{c}\text { Date of CFP } \\
\mathbf{3}^{\text {rd }} \text { episode }\end{array}$ & $\begin{array}{l}\text { Duration of } \\
\text { hospitalization } \\
\text { (days) }\end{array}$ \\
\hline Cat & August 7, 2012 & 6 & September 8, 2013 & 23 & May 20, 16 Unrecorded & Und \\
Cat & April 10, 2012 & 28 & June 12, 2014 & 16 & & \\
Cat & January 7, 2013 & Unrecorded & April 18, 2013 & 12 & \\
Dog & December 27, 2011 & 42 & October 24, 2012 & 1 & \\
Dog & November 29, 2012 & 15 & February 1, 2013 & 17 & \\
Dog & June 18, 2012 & Unrecorded & September 22, 2012 & 25 & \\
Dog & May 3, 2011 & Unrecorded & June 4, 2011 & 2 & \\
Dog & August 8, 2015 & 51 & March 13, 2016 & 6 & \\
Dog & August 8, 2015 & 24 & March 14, 2016 & 11 & & \\
\hline
\end{tabular}

$\mathrm{CFP}=$ Ciguatera fish poisoning

Table-8: Duration of anorexia and recumbency in ciguatera fish poisoning cases.

\begin{tabular}{|c|c|c|c|c|c|c|}
\hline Variable & & inorexi & & & ecumb & \\
\hline Population & Combined & Dogs & Cats & Combined & Dogs & Cats \\
\hline Number of cases & 116 & 80 & 36 & 107 & 73 & 34 \\
\hline Mean & 6.1 & 6.1 & 5.9 & 11.7 & 12.5 & 10.0 \\
\hline Median & 5 & 5 & 5 & 10 & 10 & 9 \\
\hline Range & $0-32$ & $0-32$ & $0-15$ & $0-38$ & $0-38$ & $0-31$ \\
\hline
\end{tabular}

${ }_{1}^{1}$ Days anorexic=Date started eating - date presented. ${ }^{2}$ Days recumbent=Date standing/walking - date presented

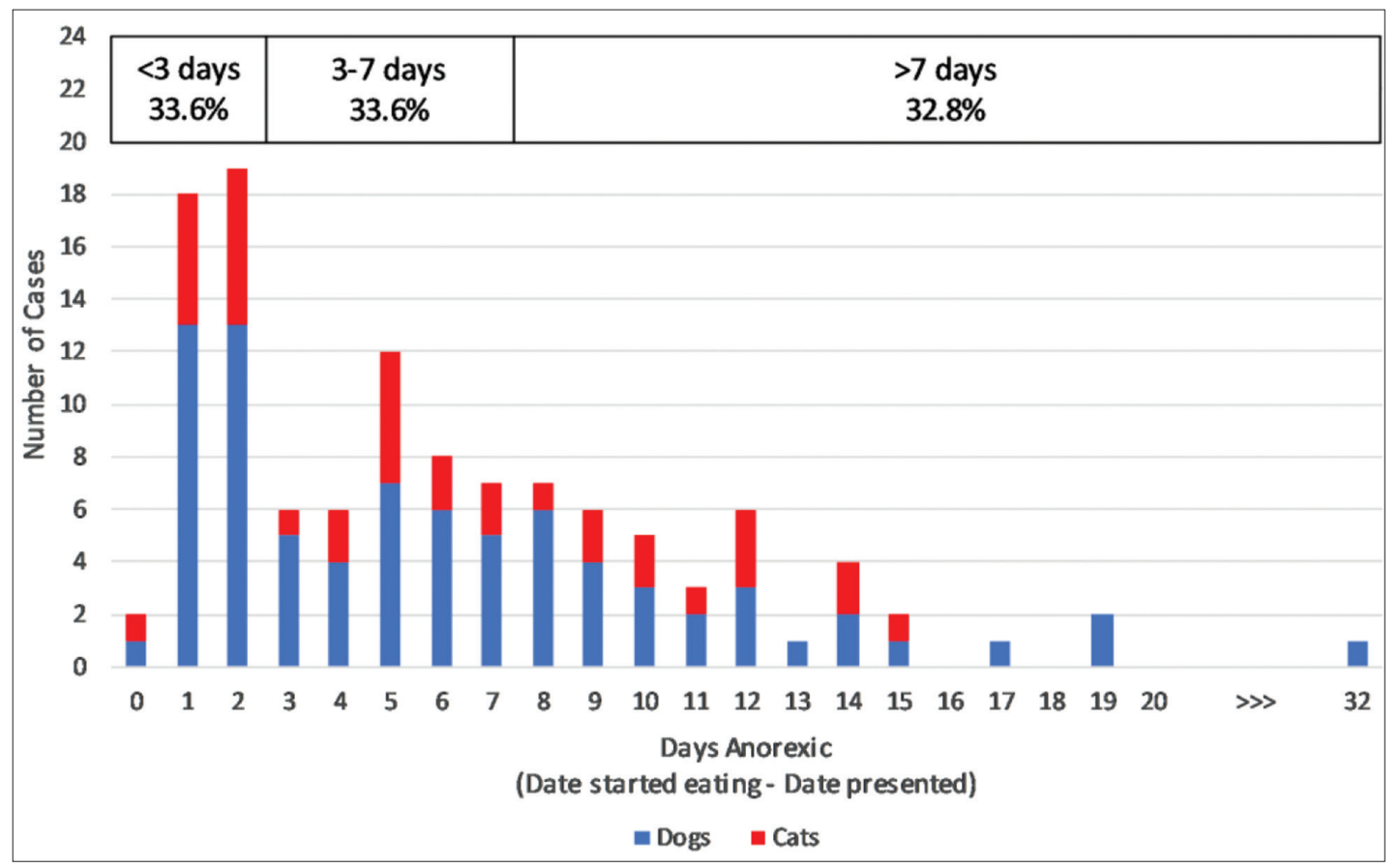

Figure-3: Duration of anorexia in canine and feline cases of ciguatera fish poisoning: Data from 116 cases with anorexia.

At the EHF Animal Clinic, cases were generally discharged once eating and walking with only mild ataxia/paresis. It is possible that depending on owner factors, some cases may have been discharged earlier or later in the recovery process.

\section{Treatment}

Decontamination was attempted in only a quarter of the study population. This may be because cases only presented after the onset of clinical signs, i.e., many hours after fish ingestion, and the window of opportunity for decontamination was perceived to have passed.
In addition, oral decontamination techniques would have been contraindicated in many cases due to the risk of aspiration (including seven cases that presented with reduced gag, 25 that presented with convulsions or opisthotonos, and 35 that were obtunded [25]). When decontamination was attempted, activated charcoal was the mainstay of therapy. The efficacy of activated charcoal in binding ciguatoxin is, however, unknown. The difficulties of decontamination, combined with the lack of a specific antidote, meant that treatment of CFP cases was primarily symptomatic. 


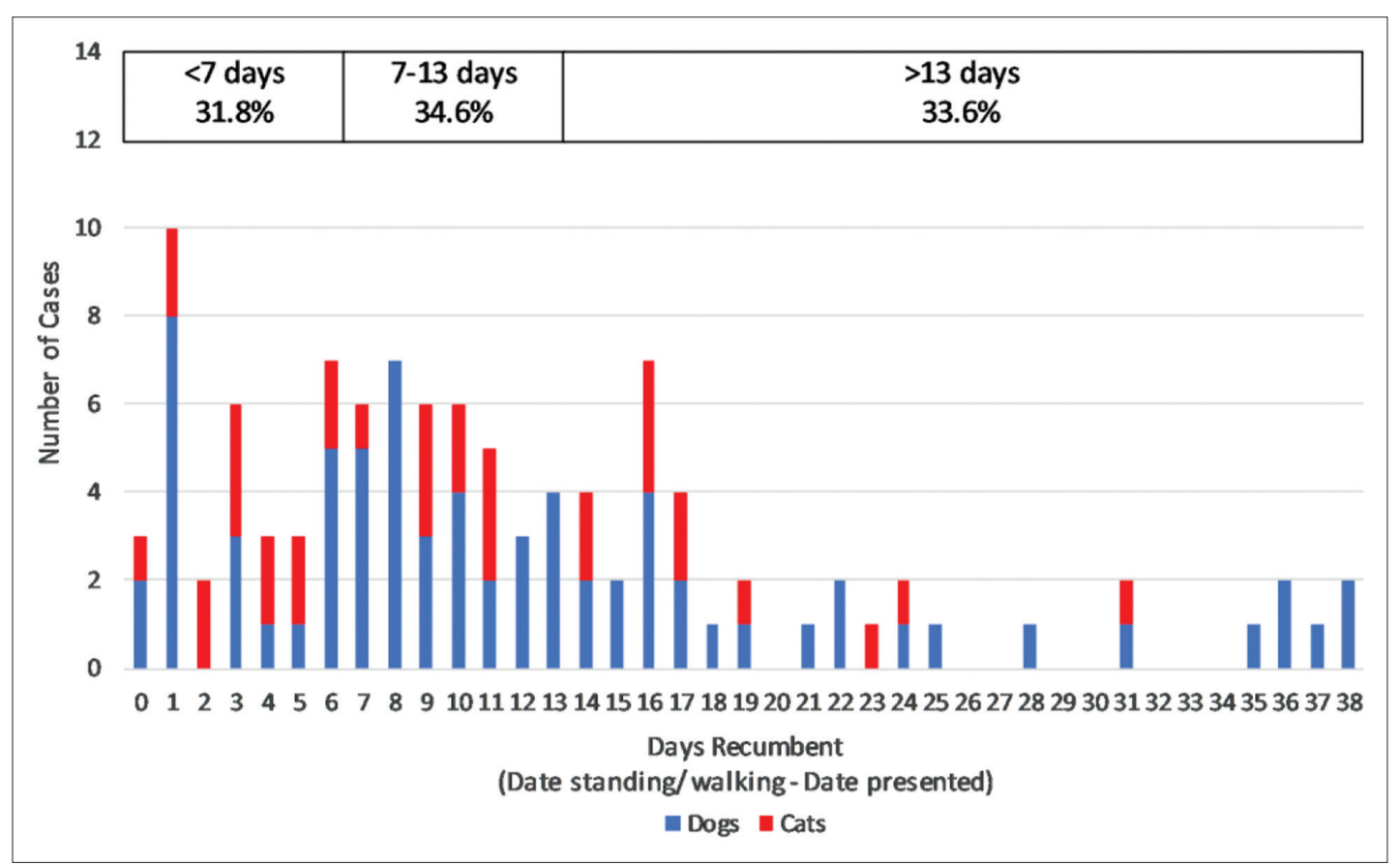

Figure-4: Duration of recumbency in canine and feline cases of ciguatera fish poisoning: Data from 107 cases with recumbency.

The frequency of therapies used in the study population generally corresponded with the frequency of the clinical signs they targeted (Supplementary Table-2). Fluid therapy and muscle relaxants were most commonly administered (Table-2). These accord with a high frequency of anorexia/dehydration and extensor rigidity/opisthotonos [25]. One discrepancy was the infrequent provision of respiratory support to dyspnoeic patients. This is likely due to a shortage of therapeutic options - oxygen supplies are limited and ventilation not possible at the EHF Animal Clinic. Another exception was nutritional support, which was only provided to $36 \%$ of cases despite $56 \%$ exhibiting inappetence or anorexia. This discrepancy may be due to animals recovering their appetite before nutritional support was deemed necessary. However, feeding tubes were only utilized in 13 cases despite 38 animals remaining anorexic for more than a week (Figure-3). Possible explanations include a shortage of clinical supplies (feeding tubes/liquid diets); anesthetic concerns (precluding esophageal tube placement); or an oversight of the attending veterinarians.

Many of the medications administered in this study have not been previously reported (except as part of the EHF Animal Clinic CFP treatment protocol [18]). This is not an indication of new developments in CFP management, but rather an indictment of the existing literature base. Gastrointestinal medications and analgesics are commonly used for the symptomatic treatment of human CFP [1], but this is their first documented use in dogs and cats. Muscle relaxants and sedatives are also reported for the first time in this study. These agents are rarely used in human CFP, but as people experience comparatively little motor dysfunction [1], the difference is not surprising.
Of the treatments that have been previously reported to treat canine and feline CFP $[4,12]$, B vitamins, atropine, and steroids were all used in this study, but only in a small number of cases.

The medications administered in this study may have been influenced by factors other than patient assessment. As a charitable foundation, the EHF Animal Clinic relies primarily on donations for stock; therefore, availability may have dictated medication choice. Consequently, no inference can be made from the prevalence of a particular drug within a therapeutic group. The CFP treatment protocol may also have influenced the treatments administered. There is, however, evidence to suggest that clinicians used their individual judgment rather than following the dictates of a protocol. Gastrointestinal medications are mentioned only briefly in the protocol [18], but were frequently administered to cases. In contrast, mannitol is recommended within the protocol, but was only administered to five animals.

The infrequent use of mannitol in the study population is surprising. Mannitol is suggested to reduce both the severity and duration of CFP symptoms in humans and is one of the primary treatments recommended [1]. Extrapolation of the treatment to animals is logical, yet it was administered to only $2 \%$ of the study population. For some cases, mannitol therapy would have been contraindicated due to dehydration. In others, the perceived window of opportunity may have elapsed, as mannitol (in humans) must be given within 48-72 $\mathrm{h}$ of fish ingestion for optimal efficacy $[2,27]$. Lack of stock is unlikely to have restricted usage (based on personal experience at the clinic). A final possibility is that veterinarians were unfamiliar with the administration of mannitol, and therefore 
reluctant to use it. Because the number of cases that received mannitol was so small, no conclusions can be made about its efficacy.

\section{Outcome}

The survival rate in this case series was high $(>90 \%)$. This tallies with the generally positive outcomes of the previous case reports of CFP, only one of which reported fatalities [13]. The mortality rate in humans is even lower, at $<0.1 \%$ [34]. This could be due to differences in the availability and standard of medical care for humans versus animals. Alternatively, species differences in pathophysiology may result in a higher mortality rate in dogs and cats. Deaths in this study were most frequently attributed to respiratory failure, a cause of mortality also reported in human CFP [1,2]. However, dogs and cats with CFP manifest more motor dysfunction than humans [25] and therefore, the risk of respiratory muscle fatigue would be greater.

Analysis to detect factors associated with non-survival was not attempted. The small number of non-survivors was deemed insufficient for statistical testing. However, there were two points of note. First, three $(37.5 \%)$ of the eight animals euthanized, and one $(8.3 \%)$ of the 12 animals that died were described as senior, compared to an overall proportion of $4.1 \%$ seniors. Second, four $(20 \%)$ of the non-surviving cases suffered flystrike, compared with one $(0.5 \%)$ of the surviving animals. This suggests that age may be a negative prognostic indicator and that fly-strike may be a portent of impending mortality.

This study did not aim to investigate whether the phenomenon of sensitization occurs in dogs and cats; however, an observation was made during the collection of case data that may be pertinent. One dog was reported to have symptom relapses associated with the ingestion of chicken in the months following her recovery. Chicken has been documented as a food trigger for the recurrence of CFP symptoms in humans $[1,27]$. While a single, retrospective observation does not prove causation, the possibility warrants investigation given the implications for nutritional management of recovering patients.

\section{Duration of illness}

The duration of illness varied widely across the study population. Some animals were discharged the same day as presentation; however, most $(98.5 \%)$ were hospitalized. This contrasts with human CFP, where the hospitalization rate is estimated to be $5-12 \%[37,38]$. The discrepancy is most likely due to species differences in pathophysiology. The motor dysfunction seen in canine and feline CFP [25] is more likely to require inpatient management than the sensory dysfunction that predominates in human CFP [1]. Two thirds of cases were hospitalized for more than a week; $40 \%$ for more than 2 weeks, and $10 \%$ of cases were hospitalized for more than 4 weeks (Figure-2). Because the study population was treated at a charitable clinic, protracted hospitalization of cases was possible even with limited owner finances. The expense of treating cases at a private practice would likely result in "economic euthanasia" of some cases, which would lower the overall survival rate.

The duration of illness did not vary significantly between dogs and cats. This does not necessarily imply that species differences do not exist. They may simply be too small, relative to the overall high degree of variation in recovery times, to be detected in the present study.

It is unsurprising that the duration of illness was longer in more severe cases (Table-4). It is only logical that recovery would take longer when the degree of improvement required is greater. Severity scores were not however included in the predictive models for time to discharge (Tables-5 and 6). This may be because in the survival analysis, a multi-tiered variable that combined both locomotor and respiratory severity was used, rather than a binary mild/severe classification. Further research is needed to determine the relationship between case severity and recovery time.

Factors that were associated with prolonged hospitalization included senior age (dogs); unknown fish exposure (cats); and anorexia (both species). The association with age could be due to a higher frequency of comorbidities in this group; alternatively, older animals may be slower to repair/replace ciguatoxin bound sodium channels. The finding that cats with known fish ingestion recovered faster is perhaps surprising, but could simply reflect more rapid diagnosis and treatment in this population. The association between anorexia and prolonged hospitalization suggests that either anorexia is a consistent marker of severe toxicosis, or that nutrition is important for patient recovery.

A variety of other clinical signs were also included in the predictive models for time to discharge (Tables-5 and 6), presumably because they indicate more severe toxicity. The absence of dyspnea from the models is somewhat surprising as it is thought to correlate with CFP severity [22]. It may be because respiratory dysfunction in CFP is generally short-lived, and therefore does not contribute to the duration of illness. The absence of recumbency in the cat model is also unexpected. It is possible that misclassification of mildly affected cats as recumbent occurred, given the species tendency for torpor when ill. This would make the clinical sign too ubiquitous to be relevant as a risk factor.

\section{Duration of anorexia and recumbency}

Prolonged anorexia and recumbency were common in this case series. The data reported are probably slightly inflated by measurement bias. There is inevitably some delay between changes in a patient's status and its observation and documentation. Nonetheless, it is notable that of 116 animals with anorexia, two thirds were anorexic for more than 3 days, and a 
third did not eat for more than a week (Figure-3). Recumbency persisted even longer: Of 107 recumbent cases, two thirds remained that way for more than a week, and a third took more than a fortnight to regain mobility (Figure-4).

Sustained anorexia and protracted recumbency both have implications for case management. Nutritional support was examined in conjunction with other treatments (see above). Data on case management did not, however, extend to the husbandry and nursing care provided. This is a consequence of relying on medical records which seldom included details of nursing activities. Prolonged recumbency, as identified in this study, necessitates good animal husbandry. The recovery of recumbent CFP cases probably depends as much on the standard of nursing as any medication. Aspects of care including bladder management and physiotherapy have the potential to speed recovery, or if neglected, result in complications and prolonged morbidity. The importance of these therapies in determining patient outcome should not be underestimated, despite their omission from the review of treatment.

\section{Time to death or euthanasia}

The first week of hospitalization appears to be a critical period for dogs and cats with CFP. Eighteen of twenty non-survivors died or were euthanized within 7 days of presentation. One of the remaining non-survivors is thought to have died from hemorrhagic diarrhea unrelated to CFP. Thus, for those animals still alive 7 days after the onset of CFP, the prognosis for recovery appears excellent.

\section{Conclusion}

This article documented the treatment and outcome of animals afflicted by CFP in the Cook Islands. Therapy for CFP was primarily symptomatic and supportive. The overall survival rate was high $(>90 \%)$; and the first 7 days of hospitalization were identified as the critical period for case mortality. Recovery was often prolonged, requiring weeks of hospitalization for the resolution of anorexia and recumbency. Factors associated with prolonged recovery times included case severity, anorexia, and age (in dogs).

The results show that while the prognosis for CFP in dogs and cats is good, patience and persistence are often required for a successful outcome. Veterinarians and owners should be aware of this, and the potential expense incurred by prolonged recoveries, when embarking on treatment of CFP cases. Mannitol therapy has been recommended as a treatment for CFP in people, and a case-control study would be beneficial to determine if it can alleviate the symptoms and/or hasten the recovery of dogs and cats with CFP.

\section{Authors' Contributions}

MJG designed the study, collected the data, and wrote the manuscript. MCG performed the statistical tests and contributed the associated methods. Both authors read and approved the final manuscript.

\section{Acknowledgments}

The research was conducted in partial fulfillment of the requirements of a Master of Veterinary Medicine at Massey University, under the supervision of Dr. Kathy Parton. Thanks go to the Esther Honey Foundation (Cook Islands) for granting access to their medical records and permitting the use of the data contained therein, and also to Eriko Prior for her Japanese translations. This study did not receive any funding.

\section{Competing interests}

The authors declare that they have no competing interests.

\section{Publisher's Note}

Veterinary World remains neutral with regard to jurisdictional claims in published institutional affiliation.

\section{References}

1. Friedman, M.A., Fernandez, M., Backer, L., Dickey, R., Bernstein, J., Schrank, K., Kibler, S., Stephan, W., Gribble, M., Bienfang, P., Bowen, R., Degrasse, S., Flores, Quintana, H., Loeffler, C., Weisman, R., Blythe, D., Berdalet, E., Ayyar, R., Clarkson-Townsend, D., Swajian, K., Benner, R., Brewer, T.D. and Fleming, L.E. (2017) An updated review of ciguatera fish poisoning: Clinical, epidemiological, environmental, and public health management. Mar. Drugs, 15(3): 72.

2. Zlateva, S., Marinov, P., Yovcheva, M., Bonchev, G., Ivanov, D. and Georgiev, K. (2017) Ciguatera poisoning: Pacific disease, foodborne poisoning from fish in warm seas and oceans. Rev. J. IMAB, 23(1): 1474-1479.

3. Rhodes, L.L., Smith, K.F., Murray, J.S., Nishimura, T. and Finch, S.C. (2020) Ciguatera fish poisoning: The risk from an Aotearoa/New Zealand perspective. Toxins, 12(1): 50.

4. Bagnis, R. and Fevai, G. (1971) La ciguatera feline experimentale a Tahiti. Ciguatera-feline experiments in Tahiti. Rev. Med. Vet., 122(6): 629-638.

5. Bagnis, R., Chanteau, S., Chengue, E., Drollet, J.H., Lechat, I., Legrand, A., Pompon, A., Prieur, C., Roux, J. and Tetaria, C. (1985) Comparison of the Cat Bioassay, the Mouse Bioassay, and the Mosquito Bioassay to Detect Ciguatoxicity in Fish. Proceedings of the Fifth International Coral Reef Congress, Tahiti. p491-496.

6. Hessel, D.W., Halstead, B.W. and Peckham, N.H. (1960) Marine biotoxins. I. Ciguatera poison: Some biological and chemical aspects. Ann. N. Y. Acad. Sci., 90(3): 788-797.

7. Kawakubo, Y. and Kikuchi, K. (1942) Testing fish poisons on animals and report of a human case of fish poisoning in the South Seas. J. Nav. Med. Sci., 31(8): 30-35.

8. Legrand, A., Rentler, J.F. and Bagnis, R. (1979) Ciguateraeffets cardiques chez le cat et le rat intoxiques experimentalement. Ciguatera-cardiac effects of experimental intoxication on cat and rat. Rev. Med. Vet., 130(12): 1659-1667.

9. Legrand, A., Lotte, C. and Bagnis, R. (1985) Respiratory and Cardiovascular Effects of Ciguatoxin in Cats: Antagonistic Action of Hexamethonium, Atropine, Propanolol, Phentolamine, Yohimbine, Prazosin, Verapamil, Calcium and Lidocaine. Proceedings of the Fifth International Coral Reef Congress, Tahiti. p463-466.

10. Anonymous. (1987) Small animals-ciguatoxin and ciguatera toxicity. Aust. Vet. Pract., 17(4): 220.

11. Clark, L. and Whitwell, G.B. (1968) Ciguatera poisoning in 
cats in Brisbane. Aust. Vet. J., 44(2): 81.

12. Kemppainen, B., Avgeris, S. and Jones, J.B. (2004) Field cases of feline ciguatera. Compend. Contin. Educ. Pract. Vet., 26(2): 132-135.

13. Losacker, W. (1992) Ciguatera fish poisoning in the Cook Islands. SPC Ciguatera Inf. Bull., 2: 12-14.

14. Newman, A.J. (1970) A nervous syndrome in dogs responding to treatment with thiamine. N. Z. Vet. J., 18(1-2): 19.

15. Tonge, J.I., Battey, Y. and Forbes, J.J. (1967) Ciguatera poisoning: A report of two outbreaks and a probable fatal case in Queensland. Med. J. Aust., 2(24): 1088-1090.

16. Cohen, H.Y. (2015) Volunteering in paradise. Vet. Nurs. J., 22(10): 32-33

17. Dalefield, R. (2017) Gambierdiscus toxicus. In: Veterinary Toxicology for Australia and New Zealand. Elsevier, Masterton. p443-484.

18. Forster, D. (2009) Problematic pacific poisonings. Vet. Times, 39(26): 27-34.

19. Fowler, M.E. (2018) Marine zootoxins. In: Veterinary Zootoxicology. Ch. 3. CRC Press, Boca Raton. p19-37.

20. Lewis, R.J. (1987) Ciguatera (fish poisoning) with special reference to cats. In: Proceedings No. 103-Veterinary Clinical Toxicology. Post Graduate Committee in Veterinary Science, University of Sydney, Sydney. p59-61.

21. McPherson, C. (1998) Ciguatoxin and tetrodotoxin poisonings in the cat: Their diagnosis, treatment and management. Aust. Vet. Pract., 28(1): 26-35.

22. Seawright, A.A. (1982) Part 5: Miscellaneous toxicities and toxicants: Ciguatera poisoning. In: Animal Health in Australia. Vol. 2. Australian Government Publishing Service, Canberra. p263-264

23. Banner, A.H., Scheuer, P.J., Sasaki, S., Helfrich, P. and Alendert, C.B. (1960) Observations on ciguatera-type toxin in fish. Ann. N. Y. Acad. Sci., 90(3): 770-787.

24. Boucaud-Maitre, D., Vernoux, J-P., Pelczar, S., DaudensVaysse, E., Aubert, L., Boa, S., Ferracci, S. and Garnier, R. (2018) Incidence and clinical characteristics of ciguatera fish poisoning in Guadeloupe (French West Indies) between 2013 and 2016: A retrospective cases-series. Sci. Rep., 8(3095): 1-7.

25. Gray, M.J. and Gates, M.C. (2020) A descriptive study of ciguatera fish poisoning in Cook Islands dogs and cats: Exposure history, clinical signs and formulation of a case definition. Vet. World, 13(2): 372-385.

26. Chinain, M., Darius, H.T., Gatti, C.M. and Roué, M. (2016) Update on ciguatera research in French Polynesia. SPC Fish. Newsl., 150: 42-51.

27. Chinain, M., Gatti, C.M., Roué, M. and Darius, H.T. (2019) Ciguatera poisoning in French Polynesia: Insights into the novel trends of an ancient disease. New Microbes New Infect., 31(100565): 1-6.
28. Inserra, M.C., Israel, M.R., Caldwell, A., Castro, J., Deuis, J.R., Harrington, A.M., Keramidas, A., Garcia-Caraballo, S., Maddern, J., Erickson, A., Grundy, L., Rychkov, G.Y., Zimmermann, K., Lewis, R.J., Brierley, S.M. and Vetter, I. (2017) Multiple sodium channel isoforms mediate the pathological effects of Pacific ciguatoxin-1. Sci. Rep., 7(42810): 1-19.

29. Palafox, N.A., Jain, L.G., Pinano, A.Z., Gulick, T.M., Williams, R.K. and Schatz, I.J. (1988) Successful treatment of ciguatera fish poisoning with intravenous mannitol. JAMA, 259(18): 2740-2742.

30. Armstrong, P., Murray, P., Nesdale, A. and Peckler, B. (2016) Ciguatera fish poisoning. N. Z. Med. J., 129(1444): 111-114.

31. Friedman, M.A., Fleming, L.E., Fernandez, M., Bienfang, P., Schrank, K., Dickey, R., Bottein, M.Y., Backer, L., Ayyar, R., Weisman, R., Watkins, S., Granade, R. and Reich, A. (2008) Ciguatera fish poisoning: Treatment, prevention and management. Mar. Drugs, 6(3): 456-479.

32. Mullins, M.E. and Hoffman, R.S. (2017) Is mannitol the treatment of choice for patients with ciguatera fish poisoning? Clin. Toxicol., 55(9): 947-955.

33. Bravo, J., Suárez, F.C., Ramírez, A.S. and Acosta, F. (2015) Ciguatera, an emerging human poisoning in Europe. $J$. Aquac. Mar. Biol., 3(1): 1-6.

34. Chan, T.Y.K. (2016) Characteristic features and contributory factors in fatal ciguatera fish poisoning-implications for prevention and public education. Am. J. Trop. Med. Hyg., 94(4): 704-709.

35. Núñez-Vázquez, E., Almazán-Becerril, A., LópezCortés, D., Heredia-Tapia, A., Hernández-Sandoval, F., Band-Schmidt, C., Bustillos-Guzmán, J., GárateLizárraga, I., García-Mendoza, E., Salinas-Zavala, C., Cordero-Tapia, A., Núñez-Vázquez, E.J., AlmazánBecerril, A., López-Cortés, D.J., Heredia-Tapia, A., Hernández-Sandoval, F.E., Band-Schmidt, C.J., BustillosGuzmán, J.J., Gárate-Lizárraga, I., García-Mendoza, E., Salinas-Zavala, C.A. and Cordero-Tapia, A. (2018) Ciguatera in Mexico (1984-2013). Mar. Drugs, 17(1): 13.

36. Gray, M.J. (2020) A descriptive study of ciguatera fish poisoning in Cook Islands dogs and cats: Demographic, temporal and spatial distribution of cases. Vet. World, 13(1): 10-20.

37. Lehane, L. and Lewis, R.J. (2000) Ciguatera: Recent advances but the risk remains. Int. J. Food Microbiol., 61(2-3): 91-125.

38. Pennotti, R., Scallan, E., Backer, L., Thomas, J. and Angulo, F.J. (2013) Ciguatera and scombroid fish poisoning in the United States. Foodborne Pathog. Dis., 10(12): 1059-1066.

\section{$* * * * * * * *$}




\section{Supplementary Tables}

Supplementary Table-1: Variables of interest.

\begin{tabular}{ll}
\hline Treatment & Detail \\
\hline Decontamination & Method \\
Fluid therapy & Route \\
Nutritional support & Method \\
Supplements & Type \\
Mannitol & \\
Muscle relaxants & Drug \\
Sedatives & Drug \\
Analgesics & Drug \\
Antibiotics & Drug \\
Steroids & Drug \\
NSAIDs & Drug \\
Eye ointment & Type \\
Other & Drug \\
Outcome & \\
Severity of locomotor dysfunction (mild or severe) & \\
Severity of respiratory dysfunction (mild or severe) ${ }^{2}$ \\
Date presented & \\
Date started eating (if inappetent) & \\
Date started walking (if recumbent) & \\
Date discharged/died & \\
Days anorexic & \\
Days recumbent & \\
Days in hospital with CFP & \\
Outcome (survived, died, euthanized) & \\
Outcome notes & \\
\hline
\end{tabular}

${ }^{1}$ Mild=No locomotor signs, ataxia or paresis without recumbency; Severe=Sternal or lateral recumbency. ${ }^{2}$ Mild=No respiratory signs or tachypnea; Severe=Moderate or marked dyspnea; NSAIDs $=$ Nonsteroidal anti-inflammatory drugs
Supplementary Table-2: Frequency of clinical signs observed in ciguatera fish poisoning cases [25].

\begin{tabular}{lcc}
\hline Clinical signs & $\begin{array}{c}\text { Number of } \\
\text { reports }\end{array}$ & Percent \\
\hline Ataxia & 164 & 68.9 \\
Recumbency & 147 & 61.8 \\
Inappetence/anorexia & 133 & 55.9 \\
Paresis/paralysis/weakness & 116 & 48.7 \\
Hypertonus/extensor rigidity & 112 & 47.1 \\
Tachypnea/dyspnea & 109 & 45.8 \\
Unable to walk & 79 & 33.2 \\
Hindlimbs worse than forelimbs & 66 & 27.7 \\
Opisthotonos & 65 & 27.3 \\
Obtunded mentation & 63 & 26.5 \\
Groaning & 62 & 26.1 \\
Vocalization & 60 & 25.2 \\
Tremors & 59 & 24.8 \\
Nystagmus & 51 & 21.4 \\
Dehydration & 46 & 19.3 \\
Hypersalivation & 44 & 18.5 \\
Proprioceptive deficits & 44 & 18.5 \\
Lethargy & 34 & 14.3 \\
Vomiting & 27 & 11.3 \\
Diarrhea & 27 & 11.3 \\
Cardiac irregularities & 23 & 9.7 \\
Hyperesthesia/dysesthesia & 20 & 8.4 \\
Convulsions/seizures & 19 & 8.0 \\
Abdominal discomfort & 16 & 6.7 \\
No gag reflex & 16 & 6.7 \\
Lacrimation & 0 & 0.0 \\
\hline Of & &
\end{tabular}

${ }^{1}$ Of $n=238$ cases in which one or more clinical signs were documented. 